\title{
Robust Kinematic Calibration for Improving Collaboration Accuracy of Dual-arm Manipulators with Experimental Validation
}

\author{
Chentao Mao ${ }^{\mathrm{a}, *}$, Shuai $\mathrm{Li}^{\mathrm{b}}$, Zhangwei Chen ${ }^{\mathrm{a}}$, Xiang Zhang ${ }^{\mathrm{c}}$, Chao $\mathrm{Li}^{\mathrm{a}}$ \\ ${ }^{a}$ State Key Laboratory of Fluid Power and Mechatronic Systems, Zhejiang University, Hangzhou, China \\ ${ }^{b}$ College of Engineering, Swansea University, Swansea, UK \\ ${ }^{c}$ School of Computer Science and Technology, Hangzhou Dianzi University, Hangzhou, China
}

\begin{abstract}
Kinematic calibration has been widely employed for manipulators to promote their performance characteristics. For a dual-arm manipulator, most of the research attentions are paid to improving the absolute positioning accuracy. However, collaborative accuracy plays a critical role in mutual operations between the two arms. For example, in dangerous chemical experiments, the dual-arm manipulator is often demanded to grab a target object with the two hands, or re-grasp a test tube from one hand to the other, where the inferior collaborative accuracy may lead to the failure of experiments. Hence, in this paper, collaborative accuracy of dual-arm manipulators is well defined and fully considered as the objective for calibration. Robustness of the calibration is further guaranteed by minimizing the maximum distance error. The formulated problem is not a typical convex optimization, and gradient search algorithm does not work well for this problem. With researches on optimization moving forward, recent advances in nonlinear optimization are employed to seek for the solution effectively, and it is found that the minimax problem can be approximately linearized to a sequence quadratic programming (SQP) problem. Furthermore, a primal-dual subgradient algorithm is applied for solving the SQP problem with a fast local convergence. Finally, in order to verify the superiority of the proposed method, an experiment is performed on an IRB 14000 manipulator, and corresponding outcomes indicate that the RMS collaborative positioning and the orientation accuracies are significantly improved by $90.60 \%$ and $91.42 \%$. To the best of our knowledge, our method has reached the best collaborative accuracy compared with existing works [26, 19, 18].
\end{abstract}

Keywords: Dual-arm Manipulators, Kinematic Calibration, Robustness, Collaborative Accuracy, Relative Accuracy.

\section{Introduction}

It is increasingly popular that industrial manipulators replace humans to perform some boring but delicate operations, and plenty of the operations on the production line need two "hands" to complete cooperative tasks together. In recent years, dual-arm manipulators has attracted intensive researches such as the execution of intended missions based on captured human motion data [1], the re-grasping operations [2], service tasks like preparing a cup of coffee $[3]$ and so on $[4,5,6]$. To a great extent, their success is determined by the collaboration accuracy between the two "hands". During the machining and assembly process, geometrical parameter errors, such as joint zero offsets and rod length errors, are introduced in the manipulators, leading to the decline in accuracy of the end-effector [7]. Hence, it is critical to identify the accurate model parameters by kinematic calibration. This becomes even more so when manipulators interact with the environment with the lack of enough onboard sensors.

Generally, depending on whether external sensors are employed, kinematic calibration can be classified as two

\footnotetext{
*Corresponding author. E-mail address: mct@zju.edu.cn (C.Mao).
}

categories: self-calibration with constraints and calibration based on external sensors[8]. By physically constraining partial DOFs of the end-effector, in the procedure of self-calibration, geometric parameters can be identified only through reading the values of onboard sensors $[9,10,11]$. The dual-arm manipulator, iCub, closed the kinematic chain by touching the artificial skin attached to the endeffector [9]. This idea was extended to identify kinematic parameters using distance and sphere constraints [10]. Later in [11], the geometric parameters were calibrated by controlling the end-effector to reach the same command point in different poses. All of these methods have one thing in common, which is to establish the constraint equations containing the geometric parameters by the relative positioning relationship of the end-effector in different configurations. However, partial geometric parameter errors associated with the constrained DOFs is unobservable, and thus can not be identified [12]. Worse still, the self-calibration method only constrains the limited spatial region, and the error distribution of the entire workspace may not be improved $[10,11]$. In comparison, calibration based on external sensors can realize more accurate and convenient measurements in the whole workspace. With the development of measurement tech- 
Table 1: Comparisons on kinematic calibration with different methods.

\begin{tabular}{|c|c|c|c|c|c|c|}
\hline$\sharp$ & $\frac{\text { Measurement }}{\text { device }}$ & $\underline{\text { Manipulator type }}$ & $\frac{\text { Accuracy }}{\text { index }}$ & $\frac{\text { Orientation }}{\text { calibration }}$ & $\frac{\text { Optimization }}{\text { method }}$ & $\underline{\text { Objective function }}$ \\
\hline This & laser tracker & IRB 14000 & relative, & $\underline{\text { Yes }}$ & SQP, primal-dual & min $\max : \|$ distance error $\|_{2}$ \\
\hline paper & & & collaborative & & & \\
\hline$[18]$ & camera & IRB 2400, Puma-500 & absolute & No & Gauss-Newton, LM & $\underline{\min }$ : \|positioning error $\|_{2}$ \\
\hline$[19$ & laser tracker & IRB 6640 & absolute & Yes & QR decomposition & $\underline{\min : \| \text { pose error } \|_{2}}$ \\
\hline$[22]$ & laser tracker & $\mathrm{M}-710 \mathrm{iC}$ & absolute & Yes & Gauss-Newton & $\underline{\min : \| \text { pose error } \|_{2}}$ \\
\hline$[25]$ & laser tracker & measuring robot & relative & No & Gauss-Newton & $\underline{\min : \| \text { distance error } \|_{2}}$ \\
\hline 26$]$ & laser tracker & self-developed & relative & No & Gauss-Newton & min: $\|$ diatance error $\|_{2}$ \\
\hline
\end{tabular}

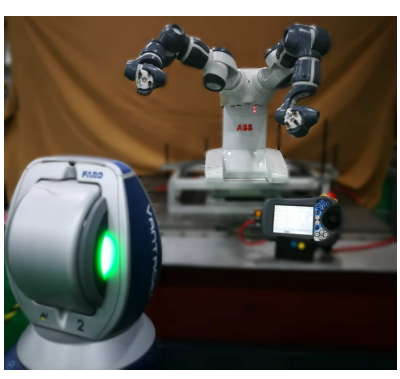

(a)

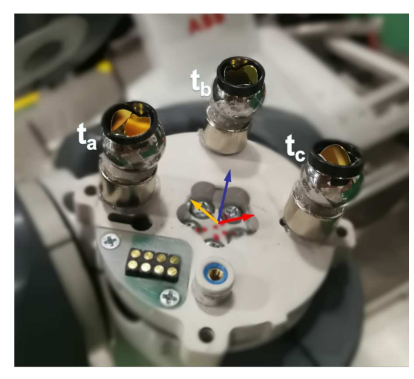

(b)
Figure 1: Experimental system for dual-arm manipulator calibration in our lab. (a) Experimental setup with an ABB IRB 14000 manipulator and a laser tracker, FARO Vantage. (b) Three end-reflectors fixed at the end-effector: $t_{a}, t_{b}$ and $t_{c}$ are the coordinates under the end effector coordinate system.

niques, there are many devices that can be used to measure the pose of manipulators, such as laser tracking systems [8, 13], three-dimensional laser scanning systems $[14,15]$, stereo visual systems with a binocular camera $[18,16,17]$ and so on. In [18], positioning accuracy was improved through minimizing the positioning errors measured by a $3 \mathrm{D}$ vision-based system. A calibration model with pose errors linearized was established in [19], where the tool coordinate system was obtained relying on the measurement of three end-points by a laser tracker. The traditional way is usually to directly establish a coordinate system from the three points to represent the pose of manipulators, which is called full pose calibration. Through a large number of experiments, it was found that the effect of the full pose calibration depends largely on what strategy is used to establish the tool coordinate system. Then, the robust search algorithm was employed in[8], by minimizing the maximum positioning error of measured end-points.

There have been some researches on the kinematic calibration of dual-arm manipulators. Exploiting the monocular camera, [20] presented an approach to estimate the kinematic parameters of a humanoid robot, Nao, where the calibration was performed on the arms and legs separately. In [21], the calibration was performed on an AR601M dual-arm robot by minimizing the absolute positioning errors of the end-effector. Both of them calibrated the single arm first, and then determined the transformation between the two arms. In previous researches, most of attentions are paid to promoting the absolute accuracy $[18,22,20,21,23,19,24]$. Nevertheless, it is quite crucial, especially for dual-arm manipulators, that an adequate collaboration accuracy between two arms should be satisfied to accomplish collaboration tasks. In this paper, a two-step calibration method will be performed like the previous researches. To promote the collaborative accuracy of dual-arm manipulators, efforts will be made from two aspects: improving the relative accuracy of the single arm as much as possible, and accurately identifying the coordinate transformation between the two arms.

For the former aspect, through large amount of experiments, it is found that the collaboration accuracy is mainly determined by the relative accuracy of the single arm itself. A kinematic model based on distance errors was presented in[25] and [26], which improved the relative positioning accuracy. However, there are few relevant researches on the relative orientation accuracy. With an inferior orientation accuracy, the relative positioning accuracy will be significantly decreased when the end-point is away from calibrated points. The problem here is how to estimate the pose of end-effectors relying on measurement of end-points, and a common method is to establish the tool coordinate system by at least three non-colinear end-points[22, 23, 24]. On one hand, there are some issues with the non-homogeneity between the residual positioning and orientation errors, and it can be solved by assigning weights of the objective function. However, the outcomes of identification is determined by the tuning weight coefficient to a considerable degree[27]. On the other hand, the values of the orientation accuracy are directly related to the reference coordinate system, which will also affect the results. In this paper, the definition of relative orientation accuracy will be introduced in the Section 2.3. Based on the distance invariance[25, 26], the residual relative errors will be minimized without the interference of coordinate transformation errors. In the meantime, both the relative positioning and orientation can be guaranteed by minimizing the maximum distance error between two configurations, 
which will be proved and experimentally verified in the Section 2.4 and 4 , respectively.

For the later aspect, [28] and [29] described in detail various sensor-robot calibration methods. However, they aimed to minimize the absolute positioning error that was represented in the measurement coordinate system. In order to ensure accurate collaborative positioning and orientation accuracy, the coordinate transformation between the two arms can also be attained by optimizing the maximum distance error between them.

The identification process can be treated as a nonlinear optimization problem. The problem has addressed a lot of attentions, and plenty of identification methods were proposed, such as Gauss-Newton method[18, 19], Levenberg-Marquardt (LM) algorithm[18], Kalman filter technique[31, 32, 33] and adaptive parameter estimation method[30] etc. With the advantage of rapid convergence, the Gauss-Newton method has been widely applied in kinematic calibration, which minimizes the sum of square residuals[19]. Then, LM algorithm solves the singularity problem that may exist in the matrix operation of GaussNewton method. In recent years, extended kalman filter method was employed in[31] to further promote the positioning accuracy. These methods are used to solve the single objective optimization problem. In this paper, to enhance the robustness, the objective function will be selected as the maximum distance error, which leads to a multi-objective problem. Thanks to the development of optimization theory in recent years, it was found that the minimax problem can be transformed into an SQP problem with a rapid convergence, thus providing a solution for the robust identification problem[8]. The comparison$\mathrm{s}$ on major characteristics of several typical methods for kinematic calibration are presented in Table 1.

The remainder of this paper is organized as follows. In Section 2, the robust kinematic calibration problem will be formulated. In Section 3, the problem described in Section 2 will be redefined as an approximate SQP problem. Section 4 will present the comparative experimental outcomes of different methods on the relative accuracy. Section 5 will present the comparative experimental outcomes of different methods on the collaborative accuracy. Section 6 will conclude this paper. Before ending this introduction section, it is worth noting that the main contributions of this paper are as follows.

- Considering characteristics of dual-arm manipulators, the scheme of collaborative accuracy is established for kinematic calibration problem, and served as a performance characteristic. Besides, the definition of collaborative accuracy and the calibration procedure can be also applied to multi-robot collaboration systems.

- Orientation accuracy is a critical aspect in robotic performance evaluation. However, common calibration methods have two apparent shortcomings of nonhomogeneity and reference coordinate system depen$\underline{\text { dency. Avoiding the above problems, a novel calibra- }}$ tion method was proposed in this paper for dual-arm manipulators by minimizing the maximum distance error to guarantee both the positioning and orientation accuracy. Besides, a minimax search algorithm is employed to enhance the robustness of the calibration procedure.

- Experimental outcomes including comprehensive comparisons validate the superiority of the proposed method for promoting the relative and collaborative accuracy, especially in terms of orientation accuracy.

\section{Robust Dual-arm Calibration Problem Formu- lation}

In this section, definitions of the collaborative and relative accuracy, the objective function and constraints are presented for optimization problem formulation. In the meantime, a novel two-step calibration method for dualarm manipulators that improves both collaborative positioning and orientation accuracy is proposed.

\subsection{Robot-Sensor System}

To achieve the goal of improving collaborative positioning and orientation accuracy, above all, the high-precision measurement system is required to measure and calculate the accuracy indicators. The experimental apparatus is set up shown in Figure 1, where the collaborative positioning and orientation accuracy is obtained by successively measuring positions of end-reflectors mounted on the end-effector in different configurations. The measuring equipment, laser tracker (FARO Vantage), with the accuracy of $16 \mu m+0.8 \mu m / m$ can achieve convenient and efficient measurement of Cartesian coordinates. Under the single-arm operation model, the end-effector successively reaches $\mathrm{k}$ arbitrary positions by manual dragging. Then, the Cartesian coordinates of all three end-reflectors are attained by the laser tracker. Simultaneously, the joint angle values are recorded from the manipulator controller, which is calculated by the corresponding motor encoder readings. In the following, a configuration of the manipulator is defined as the vector containing the coordinate values of the end-points in Cartesian space, and the corresponding joint angle values in joint space.

Dual-arm manipulators are often used to perform delicate and dangerous operations that require mutual cooperation and high precision. Therefore, the calibration method for dual-arm manipulators needs to ensure the respective operational accuracy of each arm and the accuracy of cooperation between two arms. Hence, a two-step calibration method for dual-arm manipulators is proposed, and the corresponding flow chart is shown in Figure 2. The first step is to calibrate the geometric parameters of the left and right arms separately. To solve the calibration problem defined in Section 2.6, the geometric parameters such as rod lengths and zero offsets can be updated iteratively. Exploiting the forward kinematics, the corresponding 


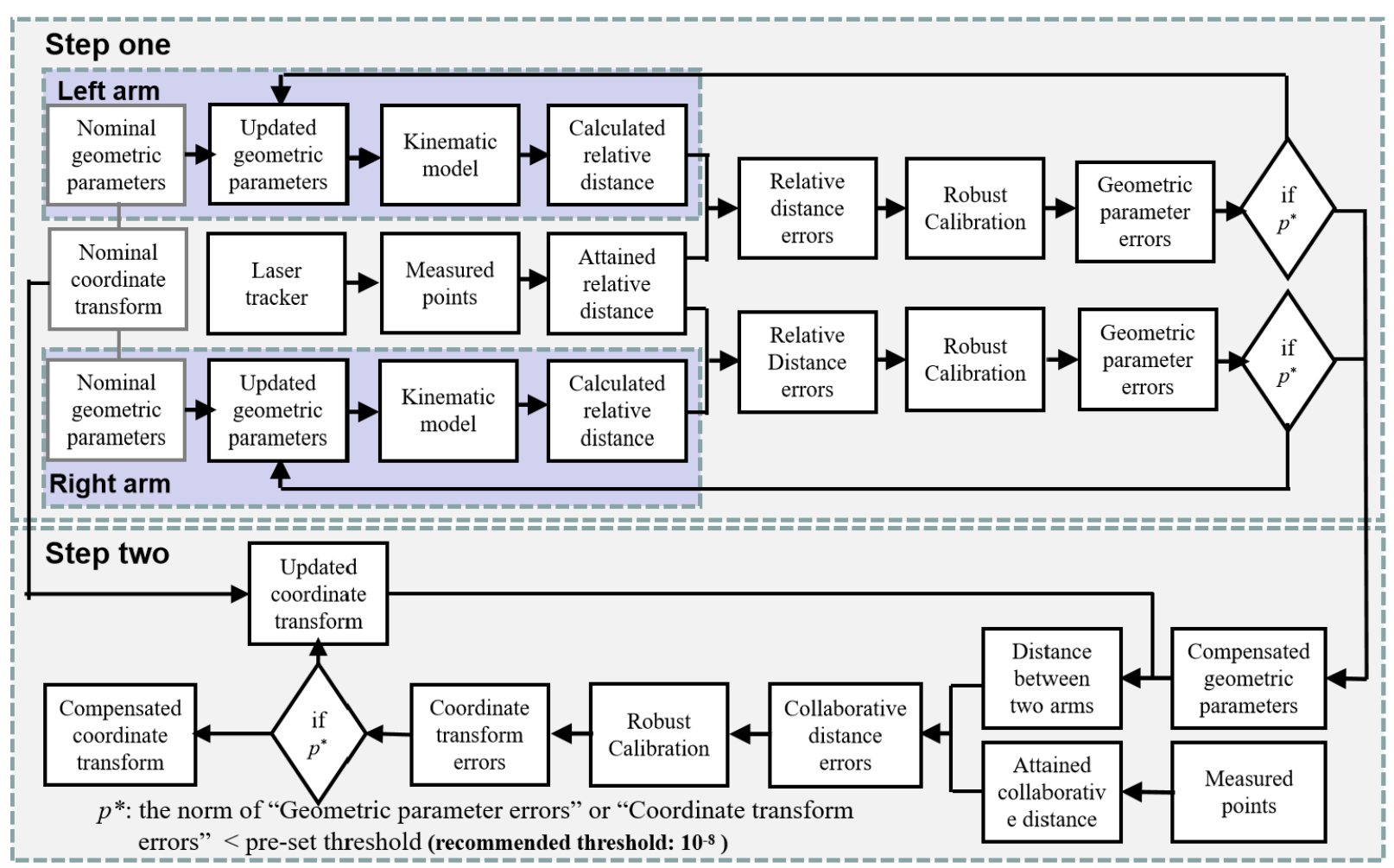

Figure 2: Flow chart of the two-step calibration method for dual-arm manipulators.

calculated relative distance is obtained by substituting the set of updated parameters. The iteration process will be terminated when the norm of identified geometric parameter errors is less than a pre-set threshold. The second step is to calibrate the coordinate transformation between the two arms after the compensation of the geometric parameters identified in step one. The calibration problem of step two will be elaborated in Section 2.7, and the iteration process is similar to the step one.

\subsection{Forward and Differential Kinematics}

Considering a dual-arm manipulator, the positions and orientations of the left and right end-effectors can be analytically represneted as following nonlinear mappings

$$
\begin{aligned}
& T_{L} \triangleq\left[\begin{array}{cc}
R_{L} & p_{L} \\
O & 1
\end{array}\right]=f\left(\theta_{L}, \mu_{L}\right), \\
& T_{R} \triangleq\left[\begin{array}{cc}
R_{R} & p_{R} \\
O & 1
\end{array}\right]=f\left(\theta_{R}, \mu_{R}\right),
\end{aligned}
$$

where the mapping $f(\cdot)$ provides the connection between the position $p \in R(3)$, the rotation matrix $R \in S O(3)$ of the end-effector coordinate in Cartesian space and the joint angle value $\theta \in R^{n}$, the geometric parameter $\mu \in R^{p \times 1}$ in the joint space. $T \in S E(3)$ is a homogeneous matrix describing the pose of the end-effector, and subscripts $L$ and $R$ indicate the left and right arms respectively. Since the two arms have the same structure and forward kinematics. For the sake of simplicity, the subscripts are omitted to indicate kinematics that are applicable to both arms. As shown in Figure 1(b), a laser tracker measures three end-reflectors fixed at the end-effector, whose coordinates are represented as $t_{a}, t_{b}$ and $t_{c} \in R(3)$. Then, the nominal coordinates of end-reflectors corresponding to the base coordinate system of manipulators can be expressed as

$$
\left[p_{a}, p_{b}, p_{c}\right]=\left[R t_{a}+p, R t_{b}+p, R t_{c}+p\right] .
$$

Take differential on both sides of (3), it can be induced that

$$
\begin{aligned}
& d p_{a}\left(\theta, \mu, t_{a}\right)=\left[J_{\mu a}, J_{t a}\right]\left[\begin{array}{l}
d \mu \\
d t_{a}
\end{array}\right], \\
& d p_{b}\left(\theta, \mu, t_{b}\right)=\left[J_{\mu b}, J_{t b}\right]\left[\begin{array}{l}
d \mu \\
d t_{b}
\end{array}\right], \\
& d p_{c}\left(\theta, \mu, t_{c}\right)=\left[J_{\mu c}, J_{t c}\right]\left[\begin{array}{l}
d \mu \\
d t_{c}
\end{array}\right],
\end{aligned}
$$

where $J_{\mu i} \in R^{3 \times p}, i=a, b$ and $c$, is the geometric parameter Jacobian matrix, reflecting the mapping from the differentiation of geometric parameters to the positions of the end-effector, and $J_{t i} \in R^{3 \times 3}, i=a, b$ and $c$, is the tool Jacobian matrix, reflecting the mapping from the differentiation of the end-point coordinates to the positions of the end-effector. Physically, the position reached is expected to be consistent with the nominal one. Unfortunately, caused by machining and assembly errors, the geometrical parameters, such as the rod lengths, zero positions of joints and so on, are deviated from the nominal values on the design drawing. Further more, deviation in joint space 
$\left(d \mu, d t_{a}, d t_{b}\right.$ and $\left.d t_{c}\right)$ leads to deviation of the end-points $\left(d p_{a}, d p_{b}\right.$ and $\left.d p_{c}\right)$, which is unevenly distributed in the Cartesian space.

\subsection{Definitions of Relative and Collaborative Accuracy}

Relative accuracy shows the ability of a serial manipulator to accurately reach the next command configuration from the current. The relative positioning accuracy of an industrial manipulator is defined in ISO 9283, namely the distance accuracy

$$
\begin{aligned}
& \Delta d=d_{m}-d_{o}, \\
& \text { where } d_{m}=\left\|p_{m i}-p_{m j}\right\|, d_{o}=\left\|p_{o i}-p_{o j}\right\|,
\end{aligned}
$$

where the subscript $m$ and $o$ indicate the measured and command values, respectively. $d_{m}$ is the distance between two attained positions, $i$ and $j$, and $d_{o}$ is the distance command, where the command positions can be readily calculated by forward kinematics of manipulators.

Since there is no clear definition of relative orientation accuracy, with reference to accuracy representation in ISO 9283, the relative orientation accuracy can be defined as

$$
R O=\left[\left|a_{m}-a_{o}\right|,\left|b_{m}-b_{o}\right|,\left|c_{m}-c_{o}\right|\right],
$$

where the relative orientation is expressed as the value of the Euler angles, $a, b$, and $c$. Unfortunately, the relative orientation defined by the Euler angles will bring about an inherent deficiency, that is, even if the orientation has only a slight change in Cartesian space, the Euler angle may vary tremendously when the Euler angles come near the singular configuration. Avoiding the problem of singularity, when the orientation error is small, a scalar indicator can be used to represent the relative orientation[24],

$$
\Delta o=\left\|\log \left(R_{m}^{\mathrm{T}} R_{o}\right)^{\vee}\right\|,
$$

where the orthogonal matrix $R$ represents the orientation transformation of the tool coordinate system from configuration $i$ to configuration $j$, and $\log \left(R_{m}^{\mathrm{T}} R_{o}\right)^{\vee} \in R^{3}$ denotes the vector representation of $\log \left(R_{m}^{\mathrm{T}} R_{o}\right) \in s o(3)$.

Collaborative accuracy reflects the ability of the two arms of dual-arm manipulators to accurately interact with each other. Inspired by the definition of relative accuracy, collaborative positioning accuracy can be defined as

$$
\begin{aligned}
& \underline{\Delta \widetilde{d}} \equiv \tilde{\bar{d}}_{\underline{m}}-\widetilde{d}_{o}, \\
& \underline{\text { where }} \widetilde{\widetilde{d}}_{m}=\| p_{L m l}-\underline{p_{R m r}\left\|, \tilde{d}_{o}=\right\| p_{L o l}-p_{\text {Ror }} \|,}
\end{aligned}
$$

where $\widetilde{d}$ denotes the distance between the two arm, and the sequence numbers of the configurations corresponding to the left and right arms are denoted as subscripts $l$ and $r$, respectively. In the same way, collaborative orientation accuracy can be defined as

$$
\Delta \widetilde{o}=\left\|\log \left(\widetilde{R}_{m}^{\mathrm{T}} \widetilde{R}_{o}\right)^{\vee}\right\|,
$$

where matrix $\widetilde{R}$ represents the orientation transformation from the end-effector of the left arm to the right arm.

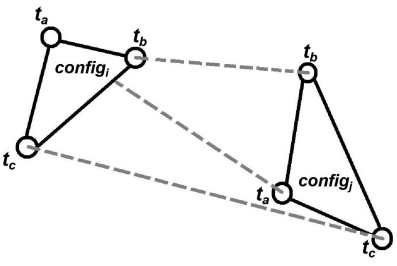

(a)

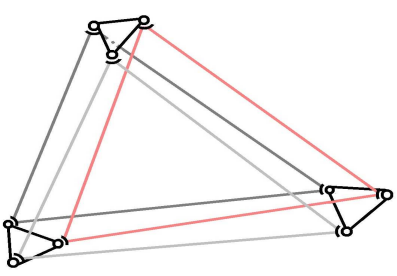

(c)

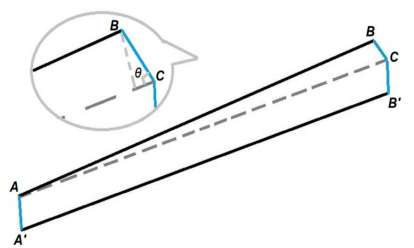

(b)

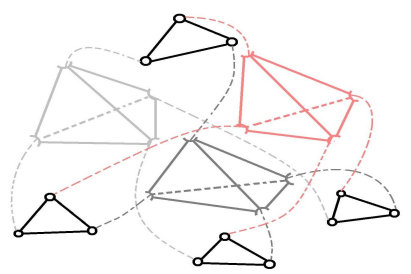

(d)
Figure 3: Schematic diagram of relative positioning and orientation optimization targets. (a) Geometric optimization targets of the relative positioning and orientation: the relative accuracy is guaranteed by minimizing the maximum distance error of the same end-reflector between two configurations, $i$ and $j$. (b) Approximate linear representation of the distance error between two end-points. (c) Geometric optimization targets among three configurations. (d) Geometric optimization targets among four configurations.

\subsection{Sufficient Condition of Relative Pose Invariance}

It is worth noting that the end-effector sensor module consisting of three end-reflectors can be regarded as a whole as shown in Figure 3(a), which represents the six dimensional motion of manipulators. Abstractly, the positioning and orientation of the end-effector can be considered as a triangle in the Cartesian space with 6 DOFs, and the relative pose between two different configurations can be constrained by two relatively invariant congruen$\mathrm{t}$ triangles. Hence, if the internal DOFs of all triangles are less than or equal to zero, the relative pose between any two configurations is invariable, which is relative pose invariance.

As shown in Figure 3(a), the corresponding corner points of the two triangles are constrained by three measured distances, and the distance constraint of each corner point between two configurations can be seen as a rod, which has two spherical joints at its extremes connecting to each corner point. In this way, a virtual mechanism is constructed to analyze the DOFs. According to the kinematic geometry theory of mechanisms, in the absence of common constraints, the DOFs of the virtual mechanism can be calculated by the Kutzbach Grübler formula with spatial mechanism[34],

$$
M=6(n-g-1)+\sum_{i=1}^{g} f_{i},
$$

where $n$ is the number of mechanical components belong- 
Table 2: DOFs of the virtual mechanical system corresponding to different numbers of configurations.

\begin{tabular}{cccc}
\hline Configs & $n$ & $g$ & $M$ \\
\hline 2 & 5 & 6 & 6 \\
3 & 6 & 9 & 3 \\
4 & 7 & 12 & 0 \\
$\mathrm{k}$ & $3+k$ & $3 k$ & $12-3 k$ \\
\hline
\end{tabular}

ing to the mechanism. $g$ is the number of kinematic pairs in the mechanism, and $f_{i}$ indicates the degrees of freedom of the $i$-th kinematic pair, here $f_{i}=3$ for the spherical pair. As shown in Figure 3(c) and 3(d), the linear rods between two configurations become triangle and tetrahedral trusses with spherical joints connecting to corresponding corner points. Since all the minimum closed loops of these trusses are triangles, the DOF of these trusses is less than or equal to zero. Then, these trusses can be seen as independent mechanical components. The DOF analysis results of this virtual mechanical system are presented in Table 2. For example, when the number of configurations is two, there are five components and six spherical pairs in the virtual mechanism as shown in Figure 3(a), and the internal DOF of the virtual mechanism is six. It can be concluded that when the number of configurations is greater than three, and the internal DOF of the virtual mechanism is less than or equal to 0 . Relative pose invariance is satisfied since the virtual mechanism is statically or super-statically determinate. Hence, it is feasible and robust to improve both relative positioning and orientation accuracy of the end-points by minimizing the maximum distance error among different configurations.

\subsection{Approximate relationship between distance and posi- tioning accuracy}

Based on the planar geometry theory, the distance error can be approximately simplified to the formula (12), which is convenient for optimization. As shown in Figure 3(b), it is assumed that the end-point $A^{\prime}$ is a nominal point in configuration $i$, and $B^{\prime}$ in configuration $j$. $A$ and $B$ are the actually attained positions measured by a laser tracker in configurations $i$ and $j$. The relationship between the distance error $\Delta d_{B}^{A}$ and the geometric parameter errors $d \mu, d t$ of manipulators can be obtained by the following analysis of plane geometry. The relative positioning accuracy between $A B$ can be written as

$$
\Delta d_{B}^{A}=|A B|-\left|A^{\prime} B^{\prime}\right|,
$$

In the meantime, the following relationship is satisfied,

$$
\begin{aligned}
\overrightarrow{A B}-\overrightarrow{A^{\prime} B^{\prime}} & =(\overrightarrow{O B}-\overrightarrow{O A})-\left(\overrightarrow{O B^{\prime}}-\overrightarrow{O A^{\prime}}\right) \\
& =\left(\overrightarrow{O B}-\overrightarrow{O B^{\prime}}\right)-\left(\overrightarrow{O A}-\overrightarrow{O A^{\prime}}\right) \\
& =\Delta \overrightarrow{p_{B}}-\Delta \overrightarrow{p_{A}}
\end{aligned}
$$

where $\Delta \overrightarrow{p_{A}}$ and $\Delta \overrightarrow{p_{B}}$ are positioning error vectors at points $A$ and $B$, respectively. The line $A C$ is parallel and equal in length to $A^{\prime} B^{\prime}$ in Figure $3(\mathrm{~b})$. Then, the connection between distances and vectors can be established by the dot product,

$$
\begin{aligned}
\overrightarrow{B^{\prime} A^{\prime}} \cdot\left(\overrightarrow{A B}-\overrightarrow{A^{\prime} B^{\prime}}\right) & =\overrightarrow{C A} \cdot \overrightarrow{C B}=|C A||C B| \cos \theta \\
& \approx|A C|(|A C|-|A B|) \\
& =-\left|A^{\prime} B^{\prime}\right| \Delta d_{B}^{A},
\end{aligned}
$$

where $|C A|>>|C B|$, hence, $|C B| \cos \theta \approx|A C|-|A B|$. Substituting formula (10) into (11), we have that

$$
\Delta d_{B}^{A} \approx \frac{\overrightarrow{A^{\prime} B^{\prime}}}{\mid A^{\prime} B^{\prime}} \cdot\left(\Delta \overrightarrow{p_{B}}-\Delta \overrightarrow{p_{A}}\right),
$$

According to the geometry theory, the distance error can be approximately expressed in the form of the absolute positioning errors of measured end-points.

\subsection{Geometric Parameter Calibration: Objective Func- tion and Nonlinear Constraint}

For geometric parameter calibration, the objective function for optimization is defined as minimizing the maximum relative positioning error, where the error can be expressed as the sum of squared distance errors corresponding to configurations $i$ and $j$, which can be expressed as

$$
\begin{aligned}
F(\mu, t) & =(\Delta d)^{\mathrm{T}} \Delta d=\sum_{i=1}^{n-1} \sum_{j=i+1}^{n}\left[\Delta d_{j}^{i}(\mu, t)\right]^{2} \\
& \approx \sum_{i=1}^{n-1} \sum_{j=i+1}^{n}\left\|\frac{\overrightarrow{P_{i}^{\prime} P_{j}^{\prime}}}{\left|P_{i}^{\prime} P_{j}^{\prime}\right|}\left(\Delta \overrightarrow{p_{j}}-\Delta \overrightarrow{p_{i}}\right)\right\|^{2}
\end{aligned}
$$

where $\Delta d$ is the sequence of distance errors. Both $\overrightarrow{P_{i}^{\prime} P_{j}^{\prime}}$ and $\left|P_{i}^{\prime} P_{j}^{\prime}\right|$ can be readily obtained by substituting the iterative nominal parameters into forward kinematics. It is worth noting that there exists an inherent constraint that the physical relative relationships among three endreflectors are invariable and independent of coordinate systems. Hence, a constrain is given that the deviation between the measured and nominal distance of any two endreflectors should be set to less than the measurement error of the laser tracker

$$
\left\{\begin{array}{l}
t_{1}^{2}=\left[\left\|t_{a}-t_{b}\right\|-\frac{1}{n} \sum_{j=1}^{n}\left\|p_{a m j}-p_{b m j}\right\|\right]^{2} \leq \epsilon, \\
t_{2}^{2}=\left[\left\|t_{b}-t_{c}\right\|-\frac{1}{n} \sum_{j=1}^{n}\left\|p_{b m j}-p_{c m j}\right\|\right]^{2} \leq \epsilon, \\
t_{3}^{2}=\left[\left\|t_{c}-t_{a}\right\|-\frac{1}{n} \sum_{j=1}^{n}\left\|p_{c m j}-p_{a m j}\right\|\right]^{2} \leq \epsilon,
\end{array}\right.
$$

where $t_{l}, l=1,2,3$ is the distance deviation between any two end-points, and $l$ is the sequence number of the nonlinear constraint. The measured distances are obtained by averaging $\mathrm{m}$ measurements, and the threshold, $\epsilon$, is determined based on the uncertainty of distance measurements. 
For optimization problem, the inequality constraint (14) can be rewritten as

$$
c(t)=\left[\begin{array}{c}
t_{1}^{2}-\epsilon \\
t_{2}^{2}-\epsilon \\
t_{3}^{2}-\epsilon
\end{array}\right] \leq 0,
$$

As proved in the section 2.4, both the relative positioning and orientation accuracy can be guaranteed by minimizing the maximum distance error, where the robustness of kinematic calibration can also be enhanced by introducing a minimax search algorithm. So the objective function with constraints can be expressed as

$$
\begin{aligned}
& \min _{\mu, t} \max _{i}: F_{i}(\mu, t), \\
& \text { subject to: } c(t) \leq 0 \text {, }
\end{aligned}
$$

where the subscript $i$ indicates the $i$-th end-reflector. By searching the optimal geometric parameters $\mu$, the objective function can be minimized.

\subsection{Coordinate Transformation Calibration: Objective Function}

The collaborative accuracy is embodied in the positioning and orientation accuracy between the end-effectors of the two arms, and the collaborative accuracy can also be guaranteed by minimizing the maximum collaborative positioning error. By the above objective function, coordinate transformation between the two arms can be identified, and the homogeneous coordinate transformation from the left arm base to the right is assumed as

$$
B=\left[\begin{array}{cc}
R_{B} & p_{B} \\
O & 1
\end{array}\right] \in S E(3) .
$$

In order to calculate the nominal distance between the left and right arms, the end-points should be represented in the same coordinate system. The end-points of the left arm can be expressed as

$$
p_{t L}=R_{L} t_{L}+p_{L},
$$

and the end-point of the right expressed in the left arm coordinate system can be deduced that

$$
p_{t R}=R_{B} R_{R} t_{R}+R_{B} p_{R}+p_{B} .
$$

Then, the collaborative distance squared error function is

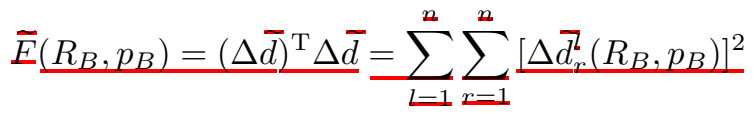

$$
\begin{aligned}
& \approx \sum_{l=1}^{n} \sum_{r=1}^{n}\left\|\frac{\overrightarrow{P_{l}^{\prime} P_{r}^{\prime}}}{\left|\overrightarrow{P_{l}^{\prime} P_{r}^{\prime}}\right|}\left(\Delta \overrightarrow{p_{R r}}-\Delta \overrightarrow{p_{L l}}\right)\right\|^{2} \\
& =\sum_{l=1}^{n} \sum_{r=1}^{n}\left\|\frac{\overrightarrow{P_{l}^{\prime} P_{r}^{\prime}}}{\mid \overrightarrow{P_{l}^{\prime} P_{r}^{\prime} \mid}} \Delta \overrightarrow{p_{R r}}\right\|^{2}
\end{aligned}
$$

where the collaborative error mainly comes from the coordinate transformation deviation embodied in $\Delta \overrightarrow{p_{R r}}$, and thus $\Delta \overrightarrow{p_{L l}}=0$. The objective function can be written as

$$
\min _{R_{B}, p_{B}} \max _{i}: \widetilde{F}_{i}\left(R_{B}, p_{B}\right),
$$

where the subscript $i$ denotes the $i$-th end-reflector. According to above analysis, the kinematic calibration of dual-arm manipulators can be converted into a minimax search optimization problem.

\section{Minimax Search Algorithm for Kinematic Cal- ibration}

In Section 2.6 and 2.7, the calibration problems have been formulated as minimax optimization problems. In this section, the minimax problems will be transformed into SQP optimization problems. Furthermore, a primaldual subgradient algorithm will be applied for solving the SQP problem with a fast local convergence.

\subsection{Redefinition of the Optimization Problem}

The minimax problems for the two-step calibration can be uniformly rewritten as an equivalent constrained optimization problem,

$$
\begin{aligned}
& \underset{x}{\operatorname{minimize}}: \psi(x), \\
& \text { subject to }: C(x) \leq 0,
\end{aligned}
$$

where $x$ is the optimizing parameters to be identified in calibration problems. For geometric parameter calibration, we have $x=(\mu, t), \psi(x)=\max _{i}\left\{F_{i}(x)\right\}$, and

$$
C(x)=\left[\begin{array}{c}
F_{i}(x)-\psi(x) \\
c(x)
\end{array}\right]
$$

For coordinate transformation calibration, they become $x=\left(R_{B}, y_{B}\right), \widetilde{\psi}(x)=\max _{i}\left\{\widetilde{F}_{i}(x)\right\}$, and $\widetilde{C}(x)=\left[\widetilde{F}_{i}(x)-\right.$ $\widetilde{\psi}(x)]$. The optimization problem described in formula (22) can be solved by generating SQP problems, which are linear approximations of the original system[35, 36]. For the sake of simplicity, the following formulas are derived with $F_{i}(x)$ as example. Owing to the differentiability of $F_{i}(x)$, then, for any direction $\Delta x$, we can have the directional derivative $D_{\Delta x} \psi(x)$,

$$
D_{\Delta x} \psi(x)=\max _{i \in I(x)}\left\{\nabla F_{i}^{\mathrm{T}}(x) \Delta x\right\},
$$

where

$$
I(x)=\left\{i: F_{i}(x)=\psi(x)\right\},
$$

Then, the approximate quadratic programming (QP) subproblem can be expressed as

$$
\begin{array}{ll}
\underset{\Delta x}{\operatorname{minimize}}: & \frac{1}{2} \Delta x^{\mathrm{T}} H_{k} \Delta x+D_{\Delta x} \psi\left(x^{k}\right), \\
\text { subject to }: & C(x)+G(x) \Delta x \leq 0,
\end{array}
$$


where the step size $\Delta x=x^{k+1}-x^{k}$, and $k$ is the number of iterations for the SQP problem. The Hessian matrix $H_{k}$ can be iteratively updated by BFGS method[37]. In addition, Karush-Kuhn-Tucker (KKT) equations should be satisfied for a constrained optimization problem, since they can provide accumulated second-order information in the Hessian matrix for a superlinear convergence. $G(x)$ is the gradient of the inequality constraints $C(x)$. Then, the Lagrangian of the KKT equations can be defined as

$$
\begin{aligned}
L(\Delta x, \lambda) \triangleq & \frac{1}{2} \Delta x^{\mathrm{T}} H_{k} \Delta x+D_{\Delta x} \psi(x) \\
& +\lambda^{\mathrm{T}}(C(x)+G(x) \Delta x),
\end{aligned}
$$

where $\lambda \geq 0$ is the Lagrange multiplier. The KKT conditions of (25) are deduced as

$$
\left\{\begin{array}{l}
\left.\nabla F_{i}(x)\right|_{i \in I(x)}+H_{k} \Delta x+\left(G^{\mathrm{T}}(x)\right)^{+} \lambda=0 \\
(C(x)+G(x) \Delta x)^{+}=0 \\
\lambda \geq 0 \\
(\lambda)^{\mathrm{T}}(C(x)+G(x) \Delta x)=0
\end{array}\right.
$$

where $(\cdot)^{+}$is defined as

$$
(x)^{+}=\left\{\begin{array}{l}
x, x \geq 0 \\
0, x<0
\end{array}\right.
$$

In order to find the optimal solution of $(\Delta x, \lambda)$, a primaldual subgradient algorithm is introduced,

$$
\left\{\begin{array}{c}
0 \in \partial_{\Delta x} L\left(\Delta x^{p}, \lambda^{p}\right)=H_{k} \Delta x^{p}+\left.\nabla F_{i}\left(x^{k}\right)\right|_{i \in I(x)} \\
+\left(G^{\mathrm{T}}\left(x^{k}\right)\right)^{+} \lambda^{p}, \\
0=-\nabla_{\lambda} L\left(\Delta x^{p}, \lambda^{p}\right)=-C\left(x^{k}\right)-G\left(x^{k}\right) \Delta x^{p},
\end{array}\right.
$$

where $\mathrm{p}$ is the number of iterations for the QP subproblem. The KKT operator is defined as

$$
T\left(\Delta x^{p}, \lambda^{p}\right)=\left[\begin{array}{c}
\partial_{\Delta x} L\left(\Delta x^{p}, \lambda^{p}\right) \\
-\left(\nabla_{\lambda} L\left(\Delta x^{p}, \lambda^{p}\right)\right)^{+}
\end{array}\right],
$$

Define $z=(\Delta x, \lambda)^{\mathrm{T}}$, and the updating law for searching the optimal $z^{*}$ is

$$
z^{p+1}=z^{p}-\alpha_{p} T^{p}
$$

where $T^{p} \in T\left(z^{p}\right)$ and $\alpha_{p}$ is the step size.

\subsection{Gradient of the Objective Function and Constraints}

An analytical gradient can give a more quick convergence than the numerical calculation of the gradient. The gradients for the objective functions of QP subproblems corresponding to the two-step calibration are derived respectively as

$$
\begin{aligned}
& P(x)=H_{k} \Delta x+\left.\nabla F_{i}(x)\right|_{i \in I(x)}, \\
& \widetilde{P}(x)=\widetilde{H}_{k} \Delta x+\left.\nabla \widetilde{F}_{i}(x)\right|_{i \in \widetilde{I}(x)},
\end{aligned}
$$

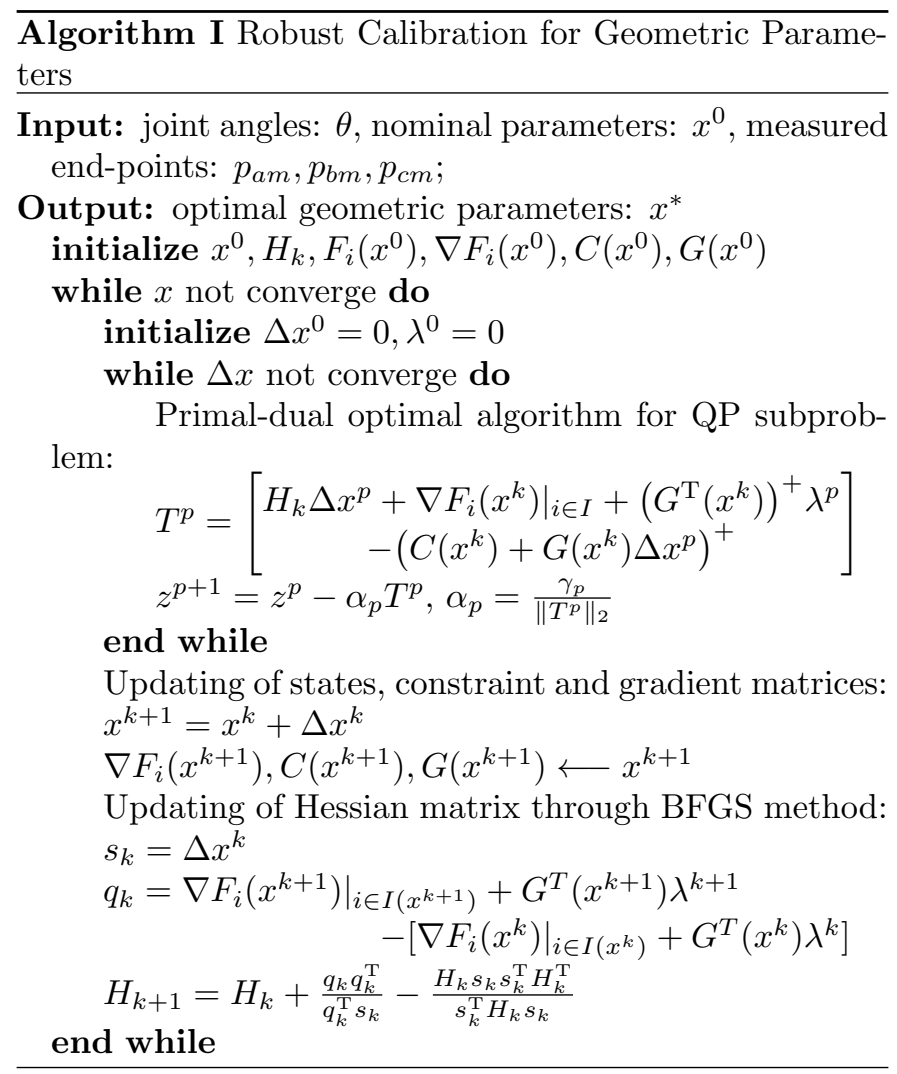

where $H_{k}$ and $\widetilde{H}_{k}$ can be obtained by BFGS method, and the gradients of $F_{i}(x)$ and $\widetilde{F}_{i}(x)$ can be deduced as

$$
\begin{aligned}
& \nabla F_{i}=2\left(\frac{\partial\left(\Delta d_{i}\right)}{\partial x^{\mathrm{T}}}\right)^{\mathrm{T}} \Delta d_{i}=2 J_{i}^{\mathrm{T}}(\theta, x) \Delta d_{i}, \\
& \nabla \widetilde{F}_{i}=2\left(\frac{\partial\left(\Delta \widetilde{d}_{i}\right)}{\partial x^{\mathrm{T}}}\right)^{\mathrm{T}} \Delta \widetilde{d}_{i}=2 \widetilde{J}_{i}^{\mathrm{T}}(\theta, x) \Delta \widetilde{d}_{i},
\end{aligned}
$$

where $J_{i}$ and $\widetilde{J}_{i}$ are the geometric parameter Jacobian matrix and the coordinate transformation Jacobian matrix corresponding to the $i$-th end-point, respectively. Exploiting the approximation of the distance error described in formula (12), the analytical expression of the two Jacobian matrices can be readily derived via differential kinematics [26]. In addition, the gradients of the constraints can be expressed as

$$
\begin{gathered}
G(x)=\left[\begin{array}{c}
\nabla F_{1}^{\mathrm{T}}(x)-\left.\nabla F_{i}^{\mathrm{T}}(x)\right|_{i \in I(x)} \\
\nabla F_{2}^{\mathrm{T}}(x)-\left.\nabla F_{i}^{\mathrm{T}}(x)\right|_{i \in I(x)} \\
\nabla F_{3}^{\mathrm{T}}(x)-\left.\nabla F_{i}^{\mathrm{T}}(x)\right|_{i \in I(x)} \\
\nabla c^{\mathrm{T}}(x)
\end{array}\right], \\
\widetilde{G}(x)=\left[\begin{array}{c}
\nabla \widetilde{F}_{1}^{\mathrm{T}}(x)-\left.\nabla \widetilde{F}_{i}^{\mathrm{T}}(x)\right|_{i \in \widetilde{I}(x)} \\
\nabla \widetilde{F}_{2}^{\mathrm{T}}(x)-\left.\nabla \widetilde{F}_{i}^{\mathrm{T}}(x)\right|_{i \in \widetilde{I}(x)} \\
\nabla \widetilde{F}_{3}^{\mathrm{T}}(x)-\left.\nabla \widetilde{F}_{i}^{\mathrm{T}}(x)\right|_{i \in \widetilde{I}(x)}
\end{array}\right],
\end{gathered}
$$

where the calculation of $\nabla F_{i}$ and $\nabla \widetilde{F}_{i}$ has been given in formula (34) and (35), and the gradient of physical con- 
$\overline{\text { Algorithm II Robust Calibration for Coordinate Trans- }}$ formation Parameters

Input: joint angles: $\theta$, nominal parameters: $x^{0}$, measured end-points: $p_{R a m}, p_{R b m}, p_{R c m}, p_{L a m}, p_{L b m}, p_{L c m}$;

Output: optimal coordinate transformation parameters: $x^{*}$

initialize $x^{0}, \widetilde{H}_{k}, \widetilde{F}_{i}\left(x^{0}\right), \nabla \widetilde{F}_{i}\left(x^{0}\right), \widetilde{C}\left(x^{0}\right), \widetilde{G}\left(x^{0}\right)$

while $x$ not converge do

initialize $\Delta x^{0}=0, \lambda^{0}=0$

while $\Delta x$ not converge do

Primal-dual optimal algorithm for QP subproblem:

$$
z^{p+1}=z^{p}-\alpha_{p} \widetilde{T}^{p} \longleftarrow \widetilde{T}^{p}
$$

end while

Updating of states, constraints, gradient matrices and

the Hessian matrix:

$x^{k+1}=x^{k}+\Delta x^{k}$

$\nabla \widetilde{F}_{i}\left(x^{k+1}\right), \widetilde{C}\left(x^{k+1}\right), \widetilde{G}\left(x^{k+1}\right), \widetilde{H}_{k+1} \longleftarrow x^{k+1}$ end while

straints, $c(x)$, is derived as

$$
\nabla c=\left[\begin{array}{l}
2 t_{1} \frac{\partial t_{1}}{\partial x} \\
2 t_{2} \frac{\partial t_{2}}{\partial x} \\
2 t_{3} \frac{\partial t_{3}}{\partial x}
\end{array}\right]=\left[\begin{array}{l}
2 t_{1} \frac{\partial\left(t_{a}-t_{b}\right)^{\mathrm{T}}}{\partial x} \frac{\left(t_{a}-t_{b}\right)}{\left\|t_{a}-t_{b}\right\|} \\
2 t_{2} \frac{\partial\left(t_{b}-t_{c}\right)^{\mathrm{T}}}{\partial x} \frac{\left(t_{b}-t_{c}\right)}{\left\|t_{b}-t_{c}\right\|} \\
2 t_{3} \frac{\partial\left(t_{c}-t_{a}\right)^{\mathrm{T}}}{\partial x} \frac{\left(t_{c}-t_{a}\right)}{\left\|t_{c}-t_{a}\right\|}
\end{array}\right],
$$

where $t=\left[t_{a}, t_{b}, t_{c}\right]^{\mathrm{T}}$ is the nominal coordinates expressed in the tool coordinate system.

\subsection{Convexity and Convergence Analysis}

Since the objective functions in Section 2.6 and 2.7 are nonlinear expressions containing trigonometric functions, the optimization problems are non-convex. However, the approximate SQP problem can be proved to be convex as follows. The objective function of the SQP problem in formula (25) is defined as

$$
Q(\Delta x)=\frac{1}{2} \Delta x^{\mathrm{T}} H_{k} \Delta x+D_{\Delta x} \psi(x),
$$

Let $\alpha$ and $\beta$ are two non-negative values, which satisfy $\alpha+\beta=1$. Then, we can yield that

$$
Q(\alpha y+\beta z)-\alpha Q(y)-\beta Q(z)=-\alpha \beta(y-z)^{\mathrm{T}} H_{k}(y-z) \leq 0,
$$

since $H_{k}$ is a positive definite matrix. According to the convex optimization theory [38], the objective function $Q(\Delta x)$ is convex. Similarly, the constraints can also be proved convex. Then, the conclusion can be drawn that the original problem converges to the local minimum with the step size [38]

$$
\alpha_{p}=\frac{\gamma_{p}}{\left\|T^{p}\right\|_{2}}, \gamma_{p}>0, \sum_{p} \gamma_{p}^{2}<\infty
$$

where $\gamma_{p}$ is a scale factor for adjusting convergence rate, and $\left\|T^{p}\right\|_{2}$ is a 2 -norm of the matrix $T^{p}$.
Table 3: Nominal DH Parameters of the IRB14000 Manipulator.

\begin{tabular}{ccccc}
\hline$\sharp$ & $a$ & $d$ & $\theta$ & $\alpha$ \\
\hline 1 & -30 & 166 & 0 & $-\pi / 2$ \\
2 & 30 & 0 & 0 & $\pi / 2$ \\
3 & 40.5 & 251.5 & 0 & $-\pi / 2$ \\
4 & 40.5 & 0 & $-\pi / 2$ & $-\pi / 2$ \\
5 & -27 & 265 & 0 & $\pi / 2$ \\
6 & 27 & 0 & 0 & $-\pi / 2$ \\
7 & 0 & 36 & 0 & 0 \\
\hline
\end{tabular}

\subsection{Algorithm for Two-step Calibration}

To summarize this section, the pseudo code of the proposed calibration method is presented in Algorithm I and II. Figure 2 has elaborately depicted the two-step calibration process for the dual-arm manipulators. Firstly, algorithm $\mathrm{I}$ is executed in the left and right arm separately and as a result, their respective geometric parameters are identified. Secondly, with the identified geometric parameters updated, algorithm II is performed to further identify the coordinate transformation between the two arms.

\section{Experimental Verification for Geometric Pa- rameter Calibration}

In this section, the geometric parameter calibration method will be experimentally validated on an IRB 14000 manipulator. It should be noted that the dual-arm manipulator is composed of two serial arms that operate separately but have the same geometric parameters, and their nominal parameters are shown in TABLE 3 .

\subsection{Numerical Validation and Analysis}

In this section, a set of arbitrary configurations for calibrating the structural parameters are selected to validate the convergence and robustness of the proposed method. In the meantime, to verify the efficacy of the calibration method, the experimental outcomes of both the configurations participating in the calibration and the additional configurations are analyzed. In the simulation, based on the rule described in Section 2.1, a total of 220 configurations are randomly generated, considering the measurement uncertainty and geometric parameter errors. These configurations are divided into two sets: one group containing 120 configurations defined as calibration configuration set, and the other consisting of the remaining 100 configurations served as verification configuration set.

As shown in Figure 4, a total of 12 calibration subsets with different number of configurations are selected from 


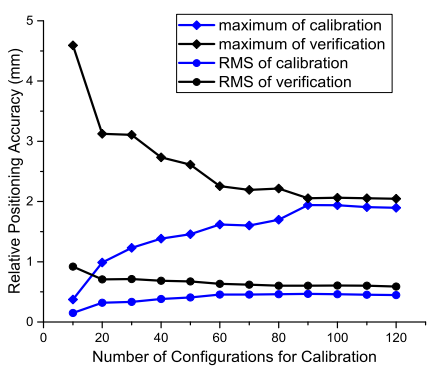

(a)

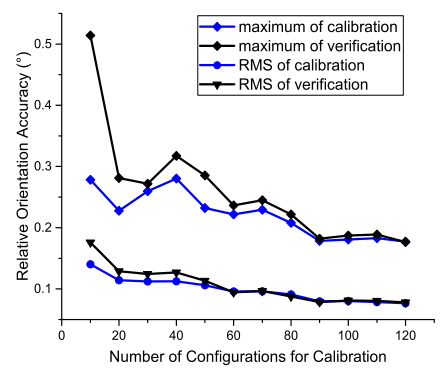

(b)
Figure 4: Convergence results for the proposed method. (a) Relative positioning accuracy changes with the increase in the number of configurations participating in calibration. (b) Relative orientation accuracy changes with the increase in the number of configurations.

the calibration configuration set, and the respective calibration results are presented. It can be seen from Figure 4(a) that, with growth of the configuration number, the relative positioning errors of the calibration subset increase, while those of the verification configuration set decrease. When the number reaches 100 , the two tend to be stable and close to each other, which means that the calibration results can improve the relative positioning accuracy in the working space even for those not participating in the calibration. What's interesting is that the relative orientation errors of both the calibration subset and the verification set are decreasing and tending to be consistent with the configuration number increasing, which is shown in Figure 4(b). It makes sense that the relative orientation accuracy is guaranteed when the maximum of the relative positioning errors of the three end-reflectors are minimized. Besides, the number of measured configurations is far beyond the required minimum for calibration, which can minimize the effects of measurement uncertainties on the calibration procedure.

\subsection{Comparisons on Relative Accuracy}

As can be seen from the analysis in Section 4.1, when enough configurations participating in the calibration are selected, the relative accuracy in the entire working space can be promoted after compensation. Hence, the 100 configurations of the left and right arms were selected respectively as shown in Figure 5, and the experimental outcomes of the proposed method in comparison to others are presented in Figure 6. It is worth noting that there exists a total of three distances between any two configurations as shown in Figure 3(a), and thus three relative positioning errors. Figure 6 indicates the distribution of relative positioning accuracy in the worst case among the three errors. It can been seen that the relative accuracy of the dualarm manipulator is extremely inferior before calibration, and there is an obvious imbalance in the positioning accuracy distribution. By contrast, after the geometric calibration, the relative errors are more likely to locate nearby

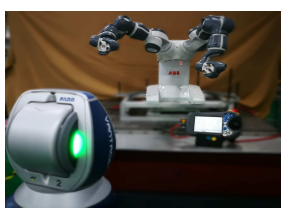

(a)

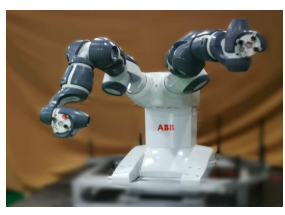

(d)

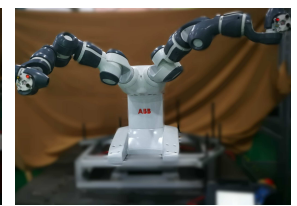

(b)

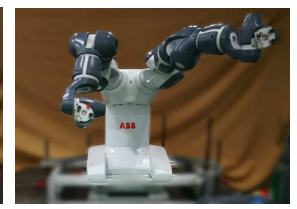

(e)

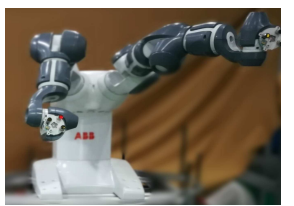

(c)

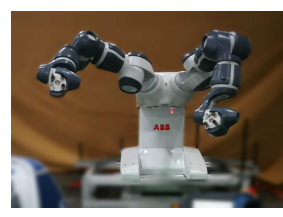

(f)
Figure 5: Measurement of end-points for robust kinematic calibration on an IRB 14000. (a) Experimental installation of measurement system. (b) Dual-arm manipulator in configuration 1. (c) Configuration 10. (d) Configuration 20. (e) Configuration 30. (f) Configuration 40.

zero. With a statistical analysis performed in Figure 6, it is found that with our method the distribution proportion of the relative positioning accuracy is $64.34 \%$ in the interval $[-0.3,0.3] \mathrm{mm}$, and $93.58 \%$ in $[-0.6,0.6] \mathrm{mm}$. Simultaneously, the distribution proportions for other methods containing both positioning and orientation accuracy are presented in Table 4 . It can be seen from Table 4 that with our method the distribution proportion nearby zero is the highest among all of the methods, which indicates our method has the best performance of the geometric parameter calibration. It is further found that the other three methods have particularly poor orientation accuracy. In particular, the method [26] has the worst orientation accuracy since it only considers minimizing the relative positioning error of one end-reflector. In Figure 6(d) and 6(h), more detailed statistics of relative accuracy is presented in the form of box diagrams.

Table 4: Frequency-distribution of relative accuracy in the specified interval.

\begin{tabular}{cccccc}
\hline Specified Interval & Nominal & Our method & {$[26]$} & {$[19]$} & {$[18]$} \\
\hline Positioning $/ \%$ & & & & & \\
{$[-0.3,0.3] \mathrm{mm}$} & 0.81 & 64.34 & 45.21 & 57.54 & 57.49 \\
{$[-0.6,0.6] \mathrm{mm}$} & 3.11 & 93.58 & 84.93 & 90.73 & 90.69 \\
\hline Orientation $/ \%$ & & & & & \\
{$[0,0.005] \mathrm{rad}$} & 2.30 & 63.96 & 13.17 & 30.10 & 29.96 \\
{$[0,0.01] \mathrm{rad}$} & 12.48 & 99.84 & 34.85 & 86.08 & 85.70 \\
\hline
\end{tabular}

RMS and maximum results of the relative accuracy are listed in Table 5. For the right arm, with our method, the RMS and maximum of positioning accuracy are promoted by $94.68 \%$ and $90.61 \%$ respectively, and the orientation accuracy are improved by $79.40 \%$ and $74.60 \%$ respectively. Other methods have improved the relative accuracy, how- 
ever, their performances are slightly more inferior than ours. The situation is different for the left arm. The experimental results of our method are better than those in the method [19] and [18], while they are almost the same as method [26]. For both our method and method [26], the RMS and maximum of positioning accuracy are promoted by $86.80 \%$ and $77.34 \%$ respectively, and the orientation accuracy are improved by $68.82 \%$ and $68.15 \%$ respectively. This is because the end-reflector selected in the method [26] is exactly the worst case among the three end-reflector, which means that the objective functions in our minimax method and the method [26] are identical.

Table 5: Comparisons on relative accuracy with different geometric parameter calibration methods.

\begin{tabular}{cccccc}
\hline Indicator & Nominal & Our method & {$[26]$} & {$[19]$} & {$[18]$} \\
\hline RIGHT ARM & & & & & \\
Positioning & $/ \mathrm{mm}$ & & & & \\
RMS $^{*}$ & 5.5609 & 0.2960 & 0.3964 & 0.3344 & 0.3347 \\
Max & 13.519 & 1.2701 & 1.4948 & 1.2587 & 1.2600 \\
Orientation & $/ \mathrm{rad}$ & & & & \\
RMS & 0.0233 & 0.0048 & 0.0142 & 0.0074 & 0.0074 \\
Max & 0.0445 & 0.0113 & 0.0253 & 0.0149 & 0.0149 \\
\hline LEFT ARM & & & & & \\
Positioning & $/ \mathrm{mm}$ & & & & \\
RMS* & 2.4538 & 0.3239 & 0.3239 & 0.3497 & 0.3494 \\
Max & 7.7423 & 1.7544 & 1.7542 & 1.7630 & 1.7432 \\
Orientation & $/ \mathrm{rad}$ & & & & \\
RMS & 0.0186 & 0.0058 & 0.0058 & 0.0075 & 0.0055 \\
Max & 0.0405 & 0.0129 & 0.0129 & 0.0167 & 0.0113 \\
\hline
\end{tabular}

* For positioning accuracy, there exists three RMS errors corresponding to the three end-reflectors. The footnote indicates that the maximum among the three RMS errors is taken.

In general, the proposed method has robust and the best performance in relative accuracy among all methods.

\section{Experimental Verification for Coordinate Transformation Calibration}

In last section, geometric parameters of the left and right arms have been identified and compensated separately, and coordinate transformation between the two arms still need to be calibrated in order to determine the collaborative relationship. In this section, the coordinate transformation calibration method will be experimentally validated on the IRB 14000 manipulator.

\subsection{Comparisons on collaborative accuracy with different coordinate transformation calibration methods}

To obtain the collaborative relationship between the two arms, a straightforward solution is to determine the coordinate transformation from the measurement coordinate system to the base of manipulators by minimizing the absolute positioning errors of measured end-points[28]. And the transformation from the left arm base to the right can be obtained by MoorePenrose inverse algorithm[39]. Inspired by geometric parameter calibration, collaborative accuracy can also be ensured by minimizing the distance error between the end-points of the two arms[26]. The experimental outcomes of the two methods are compared with ours in this section.

Table 6: Comparisons on collaborative accuracy with different coordinate transformation calibration methods.

\begin{tabular}{|c|c|c|c|c|}
\hline Indicator & Nominal & Our method & {$[26]$} & {$[28]$} \\
\hline Positioning & $/ \mathrm{mm}$ & & & \\
\hline $\mathrm{RMS}^{*}$ & 3.4126 & 0.3208 & 0.3218 & 0.3287 \\
\hline Max & 12.8463 & 1.6864 & 1.7075 & 1.5956 \\
\hline Orientation & $/ \mathrm{rad}$ & & & \\
\hline RMS & 0.0758 & 0.00650 & 0.00650 & 0.00652 \\
\hline Max & 0.1135 & 0.01370 & 0.01372 & 0.01365 \\
\hline
\end{tabular}

As presented in Figure 7 and Table 6, it can be seen that the experimental outcomes of the three methods are really close since there are only six parameters of the coordinate transformation to be tuned. Taking a close look, the maximum collaborative accuracy of our method is somewhat inferior to method [28], while our method has the smallest RMS collaborative accuracy among the three methods. With a statistical analysis of our method and [28], it is found that the distribution proportions of the collaborative positioning accuracy in the interval $[-0.3,0.3] \mathrm{mm}$ and $[-0.6,0.6] \mathrm{mm}$ are $58.53 \%$ and $91.23 \%$ with our method, respectively. With method [28], the distribution proportions are $55.78 \%$ and $91.00 \%$, respectively. Similarly, the distribution proportions of the collaborative orientation accuracy in the interval $[0,0.005] \mathrm{rad}$ and [0,0.01] rad are $33.51 \%$ and $95.34 \%$ with our method, respectively. With method [28], the distribution proportions are $31.86 \%$ and $95.20 \%$, respectively. It can be concluded that the collaborative accuracy of our method is closer to zero in comparison to method [28] from the aspect of the distribution proportion.

\subsection{Comparisons on collaborative accuracy with different geometric parameter calibration methods}

In section 5.1, it has been experimentally validated that our method with minimax search algorithm has the best performance. Then, after the coordinate transformation calibration, the experimental results of the collaborative accuracy will be demonstrated in this section. 


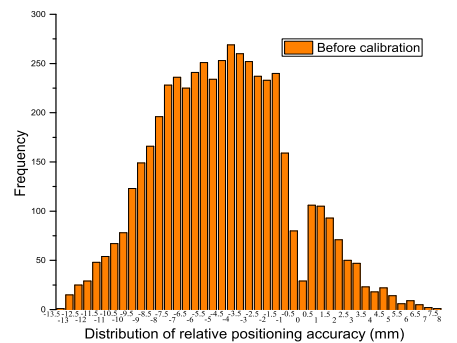

(a)

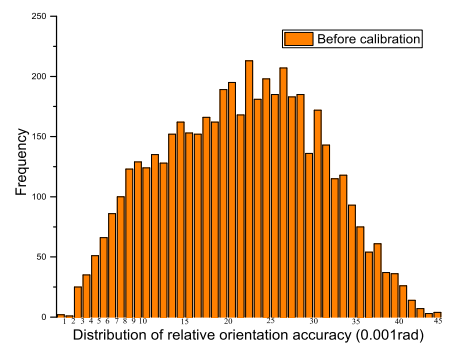

(e)

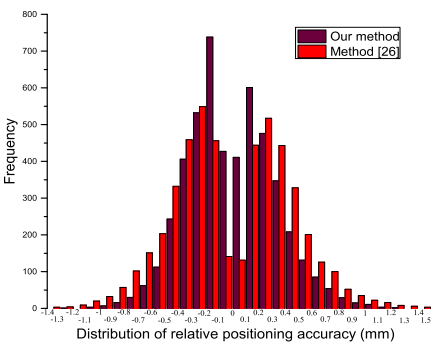

(b)

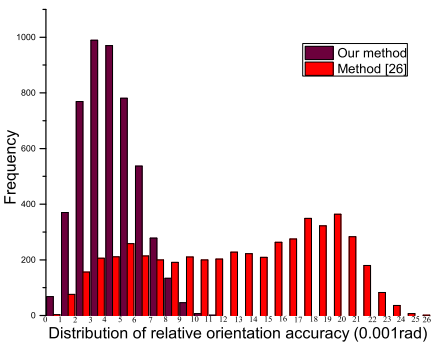

(f)

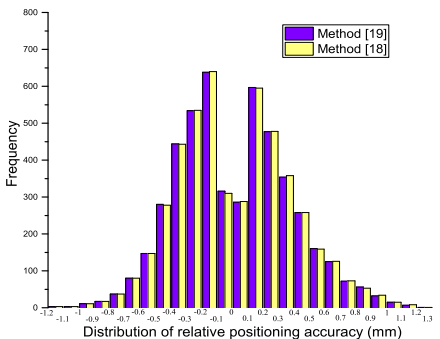

(c)

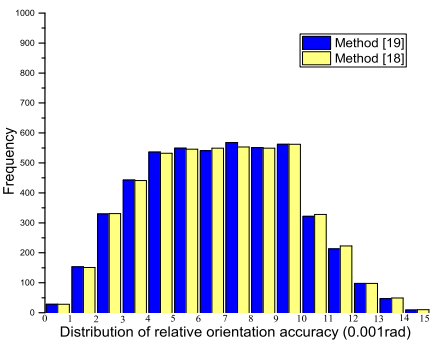

(g)

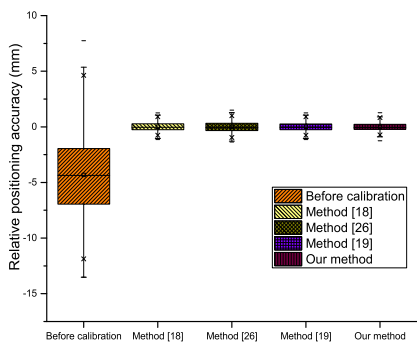

(d)

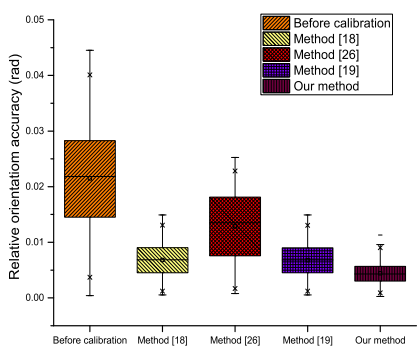

(h)

Figure 6: Comparisons on relative accuracy of the right arm with different geometric parameter calibration methods (a) Histogram of positioning accuracy before calibration. (b) Positioning accuracy between the proposed method and [26]. (c) Positioning accuracy between method [19] and [18]. (d) Box diagram of positioning accuracy. (e) Histogram of orientation accuracy before calibration. (f) Orientation accuracy between the proposed method and [26]. (g) Orientation accuracy between method [19] and [18]. (h) Box diagram of orientation accuracy. Notes: the characteristics of methods [18], [19] and [26] are elaborately described in Table 1.

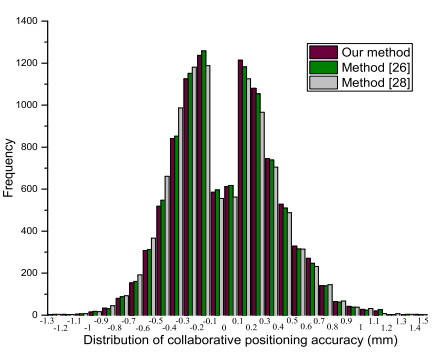

(a)

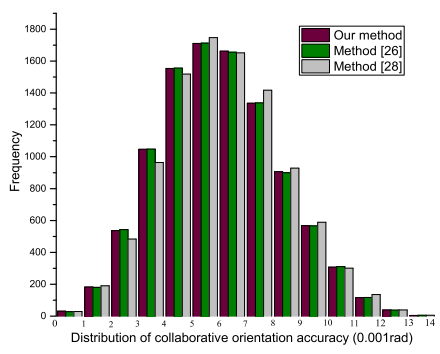

(b)
Figure 7: Comparisons on collaborative accuracy with different coordinate transformation calibration methods. (a) Positioning accuracy with the proposed method,[26] and [28]. (b) Orientation accuracy with the proposed method,[26] and [28].

Table 7: Frequency-distribution of collaborative accuracy in the specified interval.

\begin{tabular}{cccccc}
\hline Specified Interval & Nominal & Our method & {$[26]$} & {$[19]$} & {$[18]$} \\
\hline Positioning $/ \%$ & & & & & \\
{$[-0.3,0.3] \mathrm{mm}$} & 0.12 & 58.53 & 41.62 & 53.44 & 54.30 \\
{$[-0.6,0.6] \mathrm{mm}$} & 1.20 & 91.23 & 84.63 & 89.47 & 89.53 \\
Orientation $/ \%$ & & & & & \\
{$[0,0.005] / \mathrm{rad}$} & 0 & 33.51 & 4.73 & 18.99 & 16.47 \\
{$[0,0.01] / \mathrm{rad}$} & 0 & 95.34 & 30.48 & 73.08 & 72.70 \\
\hline
\end{tabular}

Figure 8 indicates the distribution of collaborative positioning accuracy in the worst case among the three errors. It can been seen that the collaborative accuracy of the dual-arm manipulator is extremely inferior before calibration. In comparison, the collaborative accuracy is closer to zero after calibration. With a statistical analysis performed in Figure 8, it is found that with our method the distribution proportion of the collaborative positioning accuracy is $58.53 \%$ in the interval $[-0.3,0.3] \mathrm{mm}$, and $91.23 \%$ in $[-0.6,0.6]$. Simultaneously, the distribution proportions for other methods containing both positioning and orientation accuracy are presented in Table 7 . It can be seen from Table 7 that with our method the distribution proportion nearby zero is the highest among all of the methods, indicating our method has the best performance. It is further found that the other three methods have particularly poor orientation accuracy. In particular, the method [26] has the worst orientation accuracy since it only considers minimizing the relative positioning error of one end-reflector. In Figure 8(d) and 8(h), more detailed statistics of relative accuracy is presented in the form of box diagrams.

RMS and maximum results of the collaborative accuracy are listed in Table 8. For the right arm, with our method, the RMS and maximum of positioning accuracy are promoted by $90.60 \%$ and $86.87 \%$ respectively, and the orientation accuracy are improved by $91.42 \%$ and $87.93 \%$ respectively. Other methods have also improved the collaborative accuracy, however, their performances are some- 


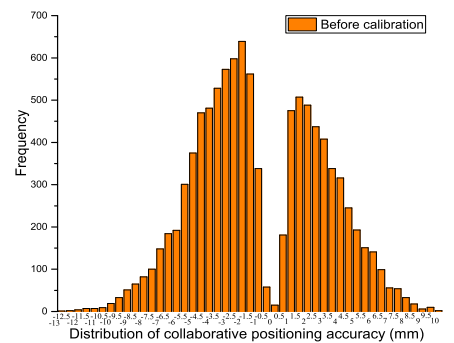

(a)

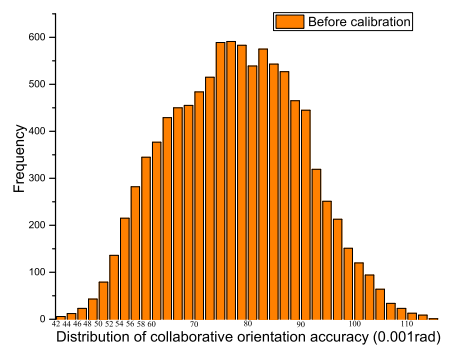

(e)

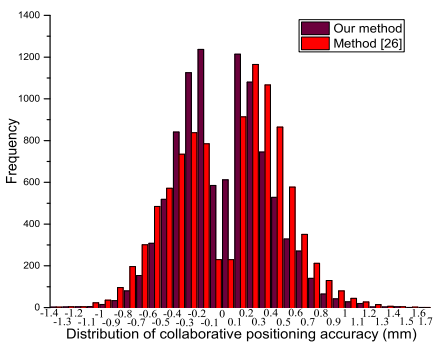

(b)

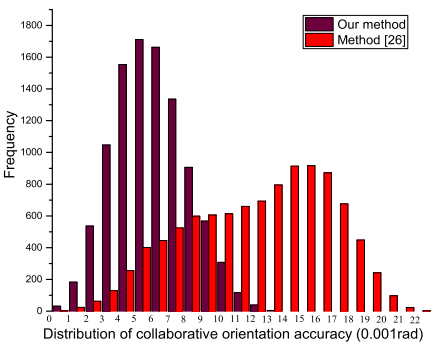

(f)

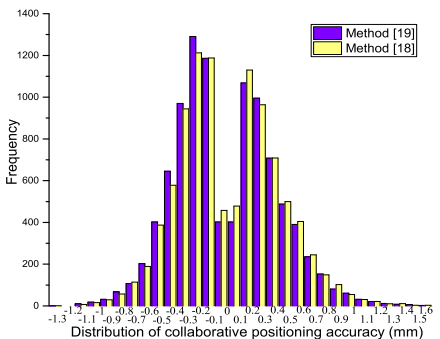

(c)

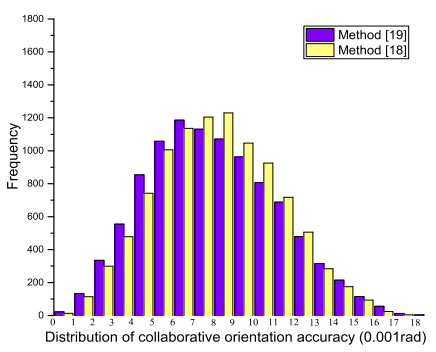

(g)

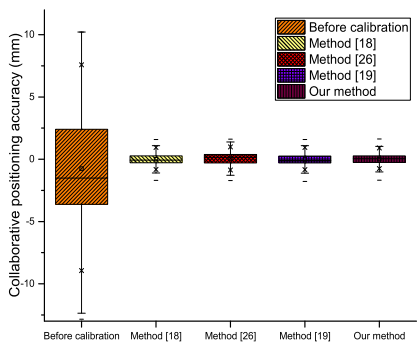

(d)

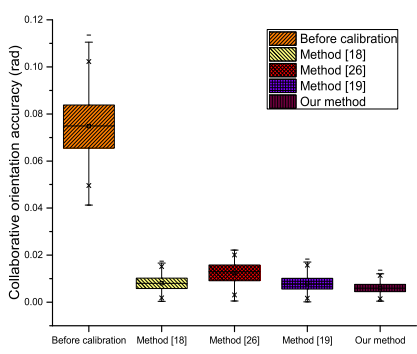

(h)

Figure 8: Comparisons on collaborative accuracy between the two arm with different geometric parameter calibration methods (a) Histogram of positioning accuracy before calibration. (b) Positioning accuracy between the proposed method and [26]. (c) Positioning accuracy between method [19] and [18]. (d) Box diagram of positioning accuracy. (e) Orientation accuracy before calibration. (f) Orientation accuracy between the proposed method and [26]. (g) Orientation accuracy between method [19] and [18]. (h) Box diagram of orientation accuracy.

what more inferior than ours. Recalling the experimental outcomes of relative accuracy in Table 5, it can be further found that the collaborative accuracy is significantly related to the relative accuracy of each single arm, especially the arm with more inferior accuracy. The collaborative positioning accuracy is close and slightly superior to the relative accuracy of the arm with more inferior accuracy. Compared with other methods in Table 6 and 8, our method promotes collaborative accuracy just a little for the coordinate transformation calibration. Nevertheless, our method of the geometric parameter calibration has overwhelming advantages over other methods.

Table 8: Comparisons on collaborative accuracy with different geometric parameter calibration methods.

\begin{tabular}{cccccc}
\hline Indicator & Nominal & Our method & {$[26]$} & {$[19]$} & {$[18]$} \\
\hline Positioning & $/ \mathrm{mm}$ & & & & \\
RMS $^{*}$ & 3.4126 & 0.3208 & 0.3758 & 0.3375 & 0.3387 \\
Max & 12.8463 & 1.6864 & 1.7026 & 1.7831 & 1.6905 \\
Orientation & $/ \mathrm{rad}$ & & & & \\
RMS & 0.0758 & 0.0065 & 0.0131 & 0.0086 & 0.0086 \\
Max & 0.1135 & 0.0137 & 0.0221 & 0.0183 & 0.0174 \\
\hline
\end{tabular}

* For positioning accuracy, there exists three RMS errors corresponding to the three end-reflectors. The footnote indicates that the maximum among the three RMS errors is taken.

In general, the proposed method has robust and the best performance in collaborative accuracy among all methods.

\section{Conclusions}

In this paper, the scheme of collaborative accuracy is established for kinematic calibration problem, and served as a performance characteristic of dual-arm manipulators. Moreover, a minimax search algorithm is introduced to promote the collaborative accuracy including positioning and orientation. The proposed method consists of two steps: geometric parameter calibration and coordinate transformation calibration. It is worth mentioning that the proposed method can be used for kinematic calibration of not only dual-arm manipulators but also cooperating robots. The novelty of this paper is listed as follows. Firstly, the relative and collaborative accuracies are ensured by minimizing the distance error. The distance error is independent on the coordinate systems and avoids the problem of non-homogeneity in [19]. Secondly, the robustness can be enhanced by a minimax search algorith$\mathrm{m}$. Finally, both the relative accuracy of single arms and collaborative accuracy between two arms are promoted significantly in comparison to other methods [26, 19, 18].

Before ending this paper, it is worth restating the accuracy improvements of dual-arm manipulators for the proposed method. Experimental outcomes on IRB 14000 robot indicates that the RMS relative positioning and the orientation accuracies are dramatically promoted by $94.68 \%$ and $79.40 \%$ respectively for the right arm, and the RMS collaborative positioning and the orientation accuracies are significantly improved by $90.60 \%$ and $91.42 \%$. Furthermore, our method has the best relative and col- 
laborative accuracy compared with the previous works $[26,19,18]$.

\section{Acknowledgement}

This work was supported by the 2017 National Key R\&D Program of China (No.2017YFB1301400).

[1] S. Shin, and C. Kim, "Human-Like Motion Generation and Control for Humanoid's Dual Arm Object Manipulation," Transactions on Industrial Electronics, vol. 62, no. 4, pp. 2265-2276, 2015.

[2] N. Vahrenkamp, D. Berenson, and T. Asfour, "Humanoid Motion Planning for Dual-Arm Manipulation and Re-Grasping Tasks," IEEE/RSJ International Conference on Intelligent Robots and Systems, 2009.

[3] B. Buml, F. Schmidt, T. Wimbck, and et al., "Catching flying balls and preparing coffee: Humanoid RollinJustin performs dynamic and sensitive tasks," IEEE International Conference on Robotics and Automation, 2011.

[4] S. Li, J. He, Y. Li, and M. Rafique, "Distributed Recurrent Neural Networks for Cooperative Control of Manipulators: A GameTheoretic Perspective," Transactions on Neural Networks and Learning System, vol. 28, no. 2, pp. 415-426, 2017.

[5] G. Hu, N. Gans, W. Dixon, "Quaternion-based visual servo control in the presence of camera calibration error," International Journal of Robust and Nonlinear Control, vol. 20, no. 5, 2010

[6] M. Khan, S. Li, Q. Wang, and Z. Shao, "Distributed Multirobot Formation and Tracking Control in Cluttered Environments," Transactions on Autonomous and Adaptive Systems, vol, 11, no. 2, pp. 1-22, 2016.

[7] Z. Roth, B. Mooring, and B. Ravani, "An Overview of Robot Calibration," Journal on Robotics and Automation, vol. 3, no. 5, pp. $377-385,1987$

[8] C. Mao, S. Li, Z. Chen, and et al., "A Novel Algorithm for Robust Calibration of Kinematic Manipulators and its Experimental Validation," IEEE Access, vol. 7, pp. 90487 - 90496, 2019.

[9] A. Roncone, M. Hoffmann, U. Pattacini, and G. Metta, "Automatic kinematic chain calibration using artificial skin: self-touch in the iCub humanoid robot," IEEE International Conference on Robotics and Automation, 2014.

[10] A. Joubair, and I. Bonev, "Kinematic calibration of a six-axis serial robot using distance and sphere constraints," The International Journal of Advanced Manufacturing Technology, vol. 77, no. 1-4, pp. 515-523, 2015.

[11] S. He, L. Ma, C. Yan, C. Lee, and P. Hu, "Multiple location constraints based industrial robot kinematic parameter calibration and accuracy assessment," The International Journal of Advanced Manufacturing Technology, 2018.

[12] G. Du, and P. Zhang, "Online robot calibration based on vision measurement," Robotics and Computer Integrated Manufacturing, vol. 29, no. 6, pp. 484-492, 2013.

[13] C. Li, Y. Wu, L. Harald, Z. Li "POE-Based Robot Kinematic Calibration Using Axis Configuration Space and the Adjoint Error Model," Transactions on Robotics, vol. 32, no. 5, pp. 1264-1279, 2016.

[14] Y. Ren, S. Yin, and J. Zhu, "Calibration technology in application of robot-laser scanning system," Optical Engineering, vol. 51, no. 11, pp. 4204, 2012.

[15] J. Li, M. Chen, and X. Jin, "Calibration of a multiple axes 3-D laser scanning system consisting of robot, portable laser scanner and turntable," Optik, vol. 122, no. 4, pp. 324-329, 2011.

[16] O. Birbach, B. Buml, and U. Frese, "Automatic and SelfContained Calibration of a Multi-Sensorial Humanoid's Upper Body," International Conference on Robotics and Automation, 2012.

[17] W. Chen, J. Du, W. Xiong, Y. Wang, S. Chia, B. Liu, and Y.Gu, "A Noise-Tolerant Algorithm for Robot-Sensor Calibration Using a Planar Disk of Arbitrary 3-D Orientation," Transactions on Automation Science and Engineering, vol. 15, no. 1, pp. 251263,2018 .
[18] J. Motta, G. Carvalho, and R. Mcmaster, "Robot calibration using a 3D vision-based measurement system with a single camera," Robotics and Computer-Integrated Manufacturing, vol.17, no. 6, pp. 487-497, 2001.

[19] X. Wang, D. Li, and M.Wang, "Complete calibration of industrial robot with limited parameters and neural network," IEEE International Symposium on Robotics and Intelligent Sensors, 2016.

20] D. Maier, S. Wrobel, and M. Bennewitz, "Whole-body selfcalibration via graph-optimization and automatic configuration selection," IEEE International Conference on Robotics and Automation, 2015.

[21] R. Khusainov, A. Klimchik, and E. Magid, "Humanoid Robot Kinematic Calibration Using Industrial Manipulator," International Conference on Mechanical, System and Control Engineering, 2017.

[22] G. Chen, H. Wang, and Z. Lin, "Determination of the Identifiable Parameters in Robot Calibration Based on the POE Formula," IEEE Transactions on Robotics, vol. 30, no. 5, pp. 1066-1077, 2014

[23] L. Wu, X. Yang, K. Chen, and H. Ren, "A Minimal POEBased Model for Robotic Kinematic Calibration With Only Position Measurements," Transactions on Automation Science and Engineering, vol.12, no. 2, pp. 758-763, 2015

[24] X. Zhang, Y. Song, Y. Yang, and H. Pan, "Stereo vision based autonomous robot calibration," Robotics Autonomous Systems, vol. 93, pp. 43-51, 2017.

[25] W. Liu, X. Qu, and Y. Yan, "Self-Calibration and Error Compensation of Flexible Coordinate Measuring Robot," IEEE International Conference on Mechatronics and Automation, 2007.

[26] Z. Wang, H. Xu, G. Chen, R. Sun and L. Sun, "A distance error based industrial robot kinematic calibration method," Industrial Robot: An International Journal, vol. 41, no. 5, pp. 439-446, 2014.

[27] Y. Wu, A. Klimchik, S. Caro, B. Furet, and A. Pashkevich, "Geometric calibration of industrial robots using enhanced partial pose measurements and design of experiments," Robotics and Computer Integrated Manufacturing, vol. 35, pp.151-168, 2015.

[28] M. Shah, R. Eastman, and T. Hong, "An Overview of RobotSensor Calibration Methods for Evaluation of Perception Systems," Proceedings of the Workshop on performance metrics for intelligent systems, 2012.

[29] S. Fanello, U. Pattacini, and I. Gori, "3D stereo estimation and fully automated learning of eye-hand coordination in humanoid robots," IEEE-RAS International Conference on $\mathrm{Hu}$ manoid Robots, 2014.

[30] J. Na, M. Mahyuddin, G. Herrmann, X. Ren, and P. Barber, "Robust adaptive finitetime parameter estimation and control for robotic systems," International Journal of Robust and Nonlinear Control, vol. 25, no. 16, pp. 3045-3071, 2015.

[31] Du G, and Ping Z, "Online Serial Manipulator Calibration Based on Multisensory Process Via Extended Kalman and Particle Filters," Transactions on Industrial Electronics, vol. 61, no. 12, pp. 6852-6859, 2014.

[32] G. Du, P. Zhang, and D. Li, "Online robot calibration based on hybrid sensors using Kalman Filters," Robotics and Computer Integrated Manufacturing, vol. 31, pp. 91-100, 2015.

[33] Z. Jiang, W. Zhou, H. Li, Y. Mo, W. Ni, and Q. Huang, "A New Kind of Accurate Calibration Method for Robotic Kinematic Parameters Based on the Extended Kalman and Particle Filter," Transactions on Industrial Electronics, vol. 65, no. 4, pp. 33373345,2018

[34] G. Gogu, "ChebychevGrblerKutzbachs criterion for mobility calculation of multi-loop mechanisms revisited via theory of linear transformations," European Journal of Mechanics - A/Solids, vol. 24 , no. 3 , pp. $427441,2005$.

[35] J. Bonnans, J. Gilbert, C. Lemarechal, and C. Sagastizabal, "Numerical Optimization-Theoretical and Practical Aspects," Transactions on Automatic Control, vol. 51, no. 3, pp. 541-541, 2003.

[36] Y. Zhang, and J. Wang, "A dual neural network for convex quadratic programming subject to linear equality and inequality constraints," Physics Letters A, vol. 298, no. 4, pp. 271-278, 2002. 
[37] R. Cottle, and M. Thapa, "Linear and nonlinear optimization," New York, NY, USA: Springer, 2017.

[38] S. Boyd, and L. Vandenberghe, "Convex optimization," London, UK: Cambridge University Press, 2004.

[39] V. Katsikis, D. Pappas, A. Petralias, "An improved method for the computation of the MoorePenrose inverse matrix," Applied Mathematics and Computation, vol. 217, no. 23, pp. 9828-9834, 2011. 